\title{
A rapid response photochromic diarylethene material for rewritable holographic data storage
}

\author{
ZHANG Qi ${ }^{1}$, LI JingMing ${ }^{2}$, NIU LiHong ${ }^{1}$, CHEN ZiHui ${ }^{1}$, YANG Lin ${ }^{1}$, ZHANG ShiMan ${ }^{1}$, \\ CAO LiangCai ${ }^{2} \&$ ZHANG FuShi ${ }^{*}$ \\ ${ }^{1}$ Key Laboratory of Organic Photoelectrons \& Molecular Engineering of Ministry of Education, Department of Chemistry, Tsinghua University, \\ Beijing 100084, China; \\ ${ }^{2}$ State Key Laboratory of Precision Measurement Technology and Instruments, Tsinghua University, Beijing 100084, China
}

Received April 10, 2012; accepted June 12, 2012; published online September 7, 2012

\begin{abstract}
A novel diarylethene, namely 4,5-(2,5-dimethyl thiophene) phthalimide, was synthesized and successfully introduced to rewritable holographic data storage. Upon the alternative illumination of UV and visible light $(\lambda>400 \mathrm{~nm})$, this compound underwent rapid, reversible inter-conversion between colorless open-ring isomer and yellow-green ring-closed form in both solution and polymethyl methacrylate (PMMA) film. Subsequently, we investigated the characteristics of volume homographic recording of the diarylethene-doped PMMA film $(1 \mu \mathrm{m}$ thick). The maximum refractive index modulation $(0.87 \%)$ of the film during recording could be reached within just $120 \mathrm{~s}$ which gave the ability of fast recording. The high quality reconstruction after 50 write/erase cycles demonstrated its excellent fatigue-resistance and high resolution. All those results indicated that this molecule was a reliable fast write/erase holographic storage material.
\end{abstract}

photochromism, diarylethene, rewritable holographic data storage, optical storage

Citation: Zhang Q, Li J M, Niu L H, et al. A rapid response photochromic diarylethene material for rewritable holographic data storage. Chin Sci Bull, 2013, 58: 74-78, doi: 10.1007/s11434-012-5368-8

With the development of information science, the demands for large storage systems are increasing daily. Optical data storage was always a very important storage technology for the advantage that the reading and writing of the digital data did not require contact and was therefore wear-free [1]. The optical data-storage formats, currently on the market such as compact disc (CD), digital versatile disc (DVD), Blue-ray disc (BD) and high density DVD (HD DVD), were based on the principles of far-field optics. For those formats, the storage density therefore followed the far-field optics scaling law NA/ $\lambda^{2}$, where NA stands for numerical aperture and $\lambda$ is the wavelength of the recording light. Theoretically, the storage density could be increased by reducing $\lambda$ and increasing the NA. However, with the advent of BD, the planar data storage has already reached its upper limit $[2,3]$.

Holographic data storage used the entire volume of the

*Corresponding author (email: zhangfs@tsinghua.edu.cn) storage medium. The data could be arranged in multiple information layers, which made it provide large storage capacity easily. The density potential reaches $V / \lambda^{3}$, where $V$ denotes the recording volume and $\lambda$ is the wavelength of the recording light source. In addition, holographic memory was a defect-tolerant device since each bit of information was generated from the entirety of a holographic memory. In view of these factors, holographic volume memory was long envisioned as the next generation storage technology [4].

Photochromism was defined as a transformation of a single chemical species between two states that have different geometrical structures, absorption spectra and refractive index, etc. The change was achieved by the illumination of lights with different wavelengths. When using photochromic materials in holographic storage, the refractive index of the photochromic molecules in the film would get changed with the laser irradiation. The holographic diffrac- 
tion efficiency mainly came from the refraction index change of the medium. With this feature, it was possible to use the interference field of content light and reference light form the refractive index modulation holographic figure in the photochromic materials film. Some successful applications have been reported [5-10].

Among all kinds of photochromic materials, organic diarylethene derivatives had been extensively studied because of their excellent fatigue resistance and thermal irreversibility [11]. However, most diarylethenes that had been applied in holographic storage suffered from several drawbacks, such as laborious synthesis procedures and rather expensive reagents. Herein, we reported a novel diarylethene, namely 4,5-(2,5-dimethyl thiophene) phthalimide, as a new type of reliable rewritable holographic storage materials. This compound was doped in polymethyl methacrylate (PMMA) thin film using the method of spin coating. The holographic recording characteristics were investigated in detail.

\section{Experimental}

\section{$1.1 \quad$ Reagents and devices}

Reagents were purchased from Beijing Chemical Reagent Company and Alfa Aesar Company. Solvents used in spectroscopy were refined by standard methods, while reagents were used as received in other experiments.

Absorption spectra were measured by using HP8452A Spectrophotometer. IR spectra were performed on a PE 2000 FT-IR Spectrometer. NMR spectra were recorded on JOEL FX-90G Spectrometer with TMS as internal reference. Mass spectroscopy was obtained on Bruker LC-MS/MS (ESQUIRE-LC) 1100 series. A Flash-EA-1112 Element Analyzer was used for elemental analyses. Photo illumination was carried out using a high pressure mercury lamp (Mejiro precision, SHG-200; 1000 W, Japan) with suitable filters.

The target molecule 4,5-(2,5-dimethyl thiophene) phthalimide $\mathbf{3}$ were synthesized according to the literature (Scheme 1) [12]. The products were characterized by NMR, IR, UV-vis, MS and elementary analysis.

\subsection{Synthesis of compound 2}

A mixture of 4,5-dibromophthalic acid dimethyl ester (3.91 g), 2,5-dimethyl-thien-3-ylboronic acid (2.96 g) and finely grounded dry potassium carbonate powder $(9.17 \mathrm{~g})$ was added into dioxane $(30 \mathrm{~mL})$ under argon flux. After being stirred for $15 \mathrm{~min}$ at room temperature, $\mathrm{Pd}\left(\mathrm{PPh}_{3}\right)_{4}(0.45 \mathrm{~g})$ was added into the argon flow. The mixture was then heated to $90^{\circ} \mathrm{C}$ for $9 \mathrm{~h}$ with TLC monitoring. After being cooled to room temperature, $\mathrm{H}_{2} \mathrm{O}(50 \mathrm{~mL})$ and $\mathrm{Et}_{2} \mathrm{O}(50 \mathrm{~mL})$ were added, and the organic and aqueous phases were separated. The aqueous layer was then extracted twice with $\mathrm{Et}_{2} \mathrm{O}$. The combined organic phase was washed with $\mathrm{NaOH}$ solution (2 $\mathrm{mol} / \mathrm{L}$ ) and pure water in turn, dried over anhydride $\mathrm{Na}_{2} \mathrm{SO}_{4}$ and concentrated. Purification by column chromatography on silica gel gave the product 4,5-bis(2,5-dimethyl thiophen-3-yl) phthalate (compound 2) (2.94 g). The total yield was $86.7 \%$.

\subsection{Synthesis of compound 3}

The compound 2 obtained from the Suzuki cross-coupling reaction $(2.8 \mathrm{~g})$ was dissolved in THF $(10 \mathrm{~mL})$ and slowly added to a $\mathrm{NaOH}$ saturated mixed solution with water (10 $\mathrm{mL})$ and methanol $(50 \mathrm{~mL})$. This was followed by stirring for $5 \mathrm{~h}$ at $40^{\circ} \mathrm{C}$. The white precipitate which formed was filtered off and washed with methanol. Then, it was dissolved in water and slowly acidified by dilute $\mathrm{HCl}(4 \mathrm{~mol} / \mathrm{L})$ at $0^{\circ} \mathrm{C}$. The yellow precipitate $(2.78 \mathrm{~g})$ was collected and dried in vacuum. The crude product obtained in the above steps was added to $\mathrm{Ac}_{2} \mathrm{O}(12 \mathrm{~mL})$ and the mixture was stirred at $80^{\circ} \mathrm{C}$ for $2.5 \mathrm{~h}$. AcOH generated in the reaction and excess $\mathrm{Ac}_{2} \mathrm{O}$ were removed by azeotropic evaporation with toluene several times, and then dried in vacuum. The product obtained above was placed in a round-bottomed flask containing $16 \mathrm{~g}$ urea under argon flow. The solid mixture was then heated to $140^{\circ} \mathrm{C}$, forming a clear, yellow melt. It was stirred for $15 \mathrm{~min}$ until bubbles ceased and then was allowed to cool to room temperature. Subsequently, water $(100 \mathrm{~mL})$ was added and the solid mixture was ultrasonically dispersed. The precipitate was collected and dried in vacuum. Purification by column chromatography on silicagel gave the target molecule 4,5-(2,5-dimethyl thiophene)

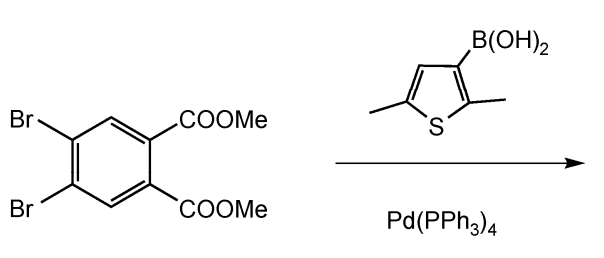

1

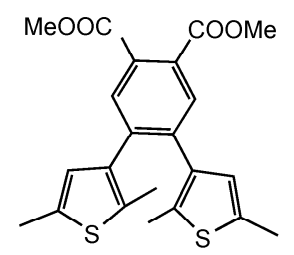

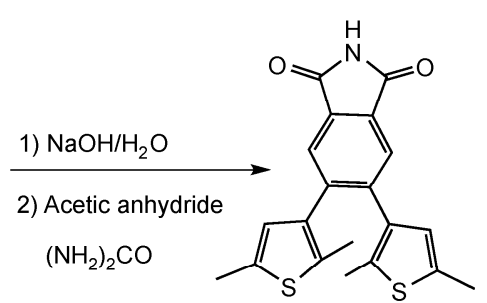

3

Scheme 1 The synthetic of target molecules 4,5-(2,5-dimethyl thiophene) phthalimide. 
phthalimide (compoud 3) $2.11 \mathrm{~g}$. The total yield was $85.3 \%$. ${ }^{1} \mathrm{H}$ NMR $\left(300 \mathrm{MHz}, \mathrm{CDCl}_{3}\right) \delta: 2.05\left(\mathrm{~s}, 6 \mathrm{H},-\mathrm{OCH}_{3}\right), 2.44$ (s, $\left.6 \mathrm{H},-\mathrm{OCH}_{3}\right), 6.27(\mathrm{~s}, 2 \mathrm{H}), 7.80(\mathrm{~s}, 2 \mathrm{H}), 7.85(\mathrm{~s}, 1 \mathrm{H}) .{ }^{13} \mathrm{C}$ NMR: 13.81, 15.14, 126.00, 127.06, 130.90, 134.27, 135.90, 136.12, 167.98. IR $\left(\mathrm{cm}^{-1}\right): 3215.99,3069.83,2953.26$, 2917.24, 2853.91, 1774.34, 1715.16, 1614.44, 1494.44, $1438.60,1372.95,1327.43,834.53,749.49$. ESI-MS: $366.17[\mathrm{M}+\mathrm{H}]^{+}$. Anal. Calcd. for $\mathrm{C}_{20} \mathrm{H}_{17} \mathrm{NO}_{2} \mathrm{~S}_{2}(\%)$ : C, 65.37; H, 4.66; N, 3.81; Found (\%): C, 65.34; H, 4.62; N, 3.82. mp: $180-182^{\circ} \mathrm{C}$.

\section{Results and discussion}

Generally, the diarylethene had two isomers, one with a less extended $\pi$-electron conjugation (open-ring isomer) and the other with a more $\pi$-electron conjugation (closed-ring isomer), and they showed distinct colors. The reversible photoisomerization of compound $\mathbf{3}$ upon the alternative illumination of UV and visible light was depicted in Scheme 2, and such process was demonstrated by UV-vis spectroscopy.

As shown in Figure 1, the solution of the open-ring isomer (3o) of compound $\mathbf{3}$ in chloroform is colorless $\left(2 \times 10^{-5}\right.$ $\mathrm{mol} / \mathrm{L}$ ), with a strong absorption at about $365 \mathrm{~nm}$. Upon the illumination of $365 \mathrm{~nm}$ light, the color of the solution turned to yellow-green. Meanwhile, two new absorption peaks at

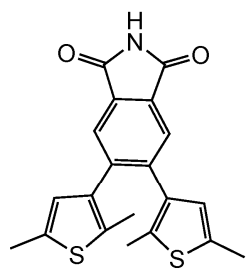

30

Open ring form

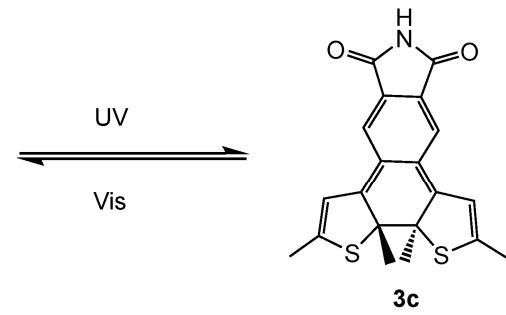

Close ring form
Scheme 2 Photoisomerization of the compound 3 upon the irradiation of different wavelength lights.

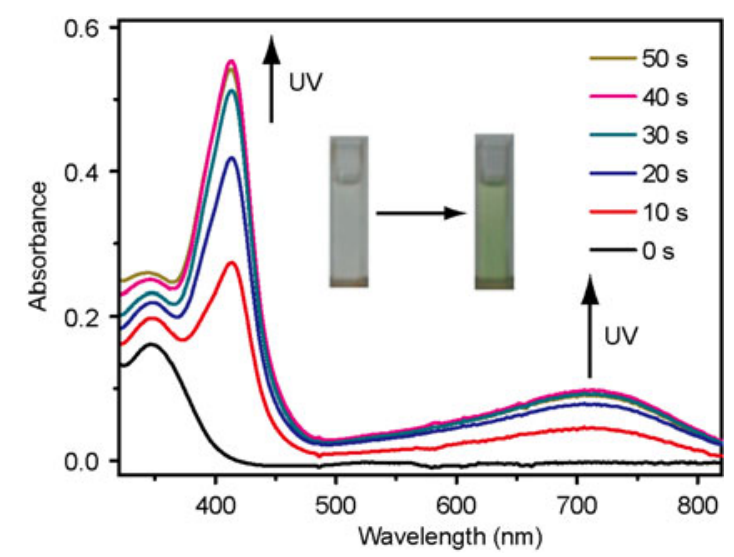

Figure 1 Absorption spectral changes of compound 3o in chloroform $\left(2.03 \times 10^{-5} \mathrm{~mol} / \mathrm{L}\right)$ with increasing UV irradiation time. Inset: the color changes of compound $\mathbf{3}$ upon UV irradiation. about 420 and $710 \mathrm{~nm}$, appeared. The photostationary state (PSS) was reached after $50 \mathrm{~s}$ and at that time, the absorbance at 420 and $710 \mathrm{~nm}$ increased to 0.55 and 0.10 respectively. The molar extinction coefficients of initial state and PSS were calculated and displayed in Table 1.

Irradiation of the PSS solution of compound $\mathbf{3}$ at the wavelength greater than $400 \mathrm{~nm}$ resulted in an immediate photo-induced ring-opening reaction which converted $\mathbf{3 c}$ to 3o. As shown in Figure 2, the original absorption spectrum was regenerated after $120 \mathrm{~s}$ and the color of the solution returned to colorless. When doped in PMMA thin film for holographic storage test, the molecule showed good fatigue-resistance. The switching process can be repeated at least 50 times and no obvious degradation was observed in the holographic storage test.

The kinetics of the photoisomerization was also studied in detail [13]. Accurate calculation in Figure 3(a) showed that $\ln (0.55-\mathrm{Abs})(0.55$ is the absorbance of PPS at $420 \mathrm{~nm}$ and Abs means the absorbance at $420 \mathrm{~nm}$ in the time of $t$ ) had a linear relationship with time which proved that this was a first order chemical reaction, the reaction rate constant $k_{1}$ is $0.025 \mathrm{~s}^{-1}$. However, in the process from PSS to 3o, the Abs just had a linear relationship with time. That meant it was a zero-order chemical reaction, and the reaction rate constant $k_{2}$ was $1.45 \times 10^{-7} \mathrm{~mol} /(\mathrm{L} \mathrm{s})$.

Subsequently, we explored the potential application of compound $\mathbf{3}$ in holographic storage. The novel diarylethene

Table 1 Molar extinction coefficient of $\mathbf{3}$ in chloroform $\left(c=2.0 \times 10^{-5}\right.$ $\mathrm{mol} / \mathrm{L}$ )

\begin{tabular}{ccc}
\hline \multirow{2}{*}{ Compound } & \multicolumn{2}{c}{$\varepsilon(\mathrm{L} /(\mathrm{mol} \mathrm{cm}))$} \\
\cline { 2 - 3 } & $420 \mathrm{~nm}$ & $710 \mathrm{~nm}$ \\
\hline 3o & 46.5 & 97.5 \\
PSS & $2.75 \times 10^{4}$ & $0.50 \times 10^{4}$ \\
\hline
\end{tabular}

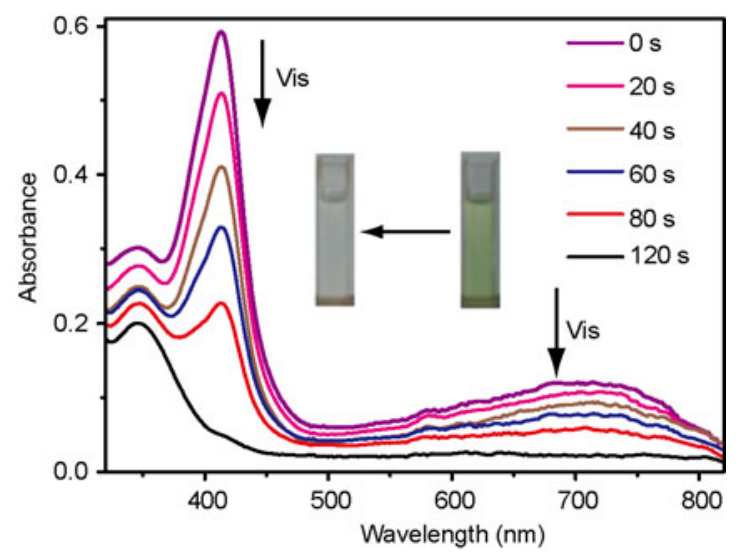

Figure 2 Absorption spectral changes of the photostationary state of compound 3 in chloroform $\left(2.15 \times 10^{-5} \mathrm{~mol} / \mathrm{L}\right)$ with increasing visible light irradiation time. Inset: the color changes of compound $\mathbf{3}$ upon visible light irradiation. 

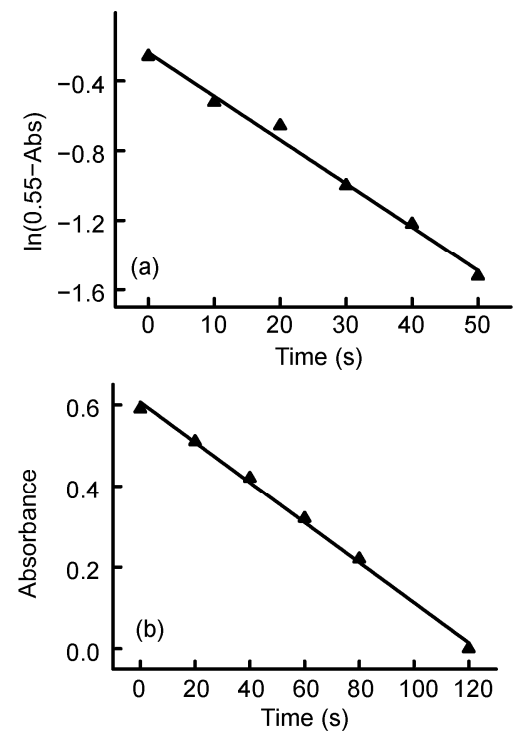

Figure 3 Kinetics of the photoisomerization. (a) From 30 to PSS upon the illumination of $365 \mathrm{~nm}$ light, first-order reaction with $k_{1}=0.025 \mathrm{~s}^{-1}$; (b) from PSS to 30 upon the illumination of light $(\lambda>400 \mathrm{~nm})$, zero-order reaction with $k_{2}=1.45 \times 10^{-7} \mathrm{~mol} /(\mathrm{L} \mathrm{s})$.

was doped in PMMA thin film using the method of spin coating [14]. The mass ratio of 3c:PMMA is $1: 4$ and the thickness was about $1 \mu \mathrm{m}$. The film was irradiated for sufficient time to ensure that $\mathbf{3 o}$ translate into $\mathbf{3 c}$ as much as possible before being used.

Figure 4 showed the optical path used to measure the diffraction efficiency and refractive index modulation of the material. A laser (532 nm) was extended, filtered collimated with a collimator filter and diaphragm. When passing through a polarized beam-splitting prism (PBS), the light was divided into two beams, a reference beam and an object beam. Two half-wave plates HP1 and HP2 were used to adjust the two beams to coherent light. Three computer operating switches were used to control the optical path and the two detectors were used to recorded the light intensity. The diarylethene-doped PMMA film was irradiated sufficient time to achieve closed-ring form. The method we used was recording for $10 \mathrm{~s}$ and measuring 10 times in a second and then take the average of 10 data. Repeat this operation to get a time-related data. When recording, the shutter 1, 2 was open, the diarylethene underwent photochemical reaction within the interference fringes of reference light and object light to form the grating. When measuring, the shutter 1,3 was open. Only the reference beam passed through the film, the detector 1, 2 separately record the diffraction intensity $I_{\mathrm{d}}$ and the transmitted light intensity $I_{\mathrm{t}}$ when the reference beam passed through the newly formed grating. Using the formula diffraction efficiency:

$$
\eta=\frac{I_{\mathrm{d}}}{I_{\mathrm{d}}+I_{\mathrm{t}}} .
$$

According to Kogelnik's two-wave coupled wave theory [15], the refractive index modulation can be expressed as

$$
\Delta n=\frac{\lambda \cos \theta \arcsin \sqrt{\eta}}{\pi d},
$$

where $d$ is the grating thickness, which was also the thickness of thin film, $\theta$ and $\lambda$ are the Bragg angle and wavelength. Using the date, the refractive index modulation during the grating recording at different times was obtained, which was shown in Figure 5. It showed us that the maximum refractive index modulation $(0.87 \%)$ could be reached

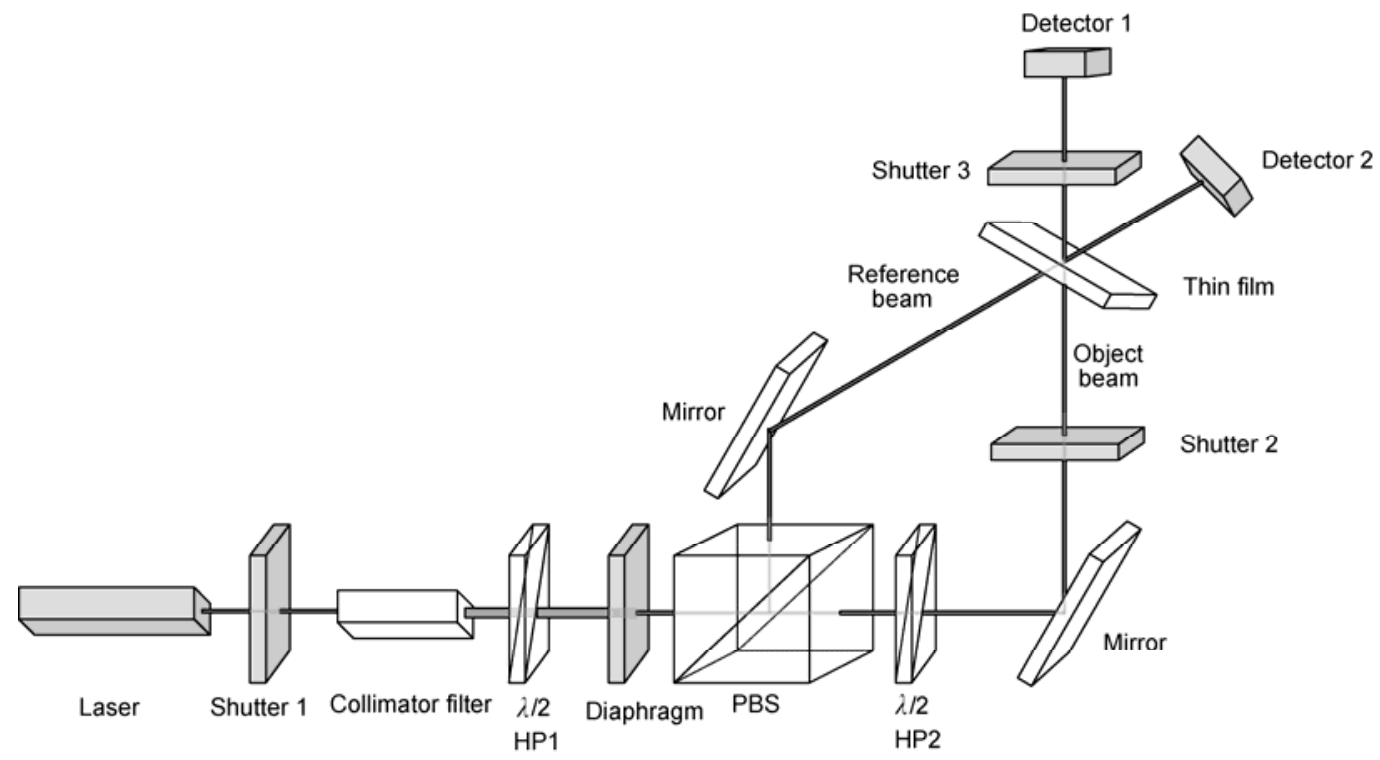

Figure 4 Experimental setup for holographic recording and readout, Grating was formed in the thin film and with the control of shutter, diffraction intensity and transmitted light intensity can be recorded by the two detectors when the reference beam passed through the film. 


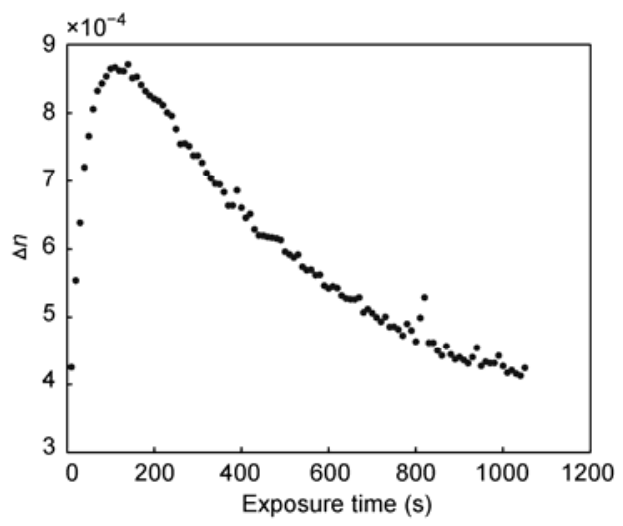

Figure 5 Refractive index modulation during holographic recording. The maximum $(0.87 \%$ ) can be reached within just $120 \mathrm{~s}$.

quickly within about $120 \mathrm{~s}$. This performance rendered the capability of fast recording of the diarylethene materials. Combined with its rapid cyclization under UV, this molecule showed its potential in fast write/erase volume holographic storage field.

When the resolution chart information was added to the object light, it would be stored on the film in the form of a grating. When the reference beam passed through the film, the resolution chart would be reconstructed. To investigate the reversible holographic storage ability of the diarylethene-PMMA film, we made a comparison between the reconstruction after first recording and the reconstruction after 50 write/erase cycles. As shown in Figure 6, there was no obvious difference between the reconstruction qualities, which indicated that this molecule was reliable rewritable holographic storage material.

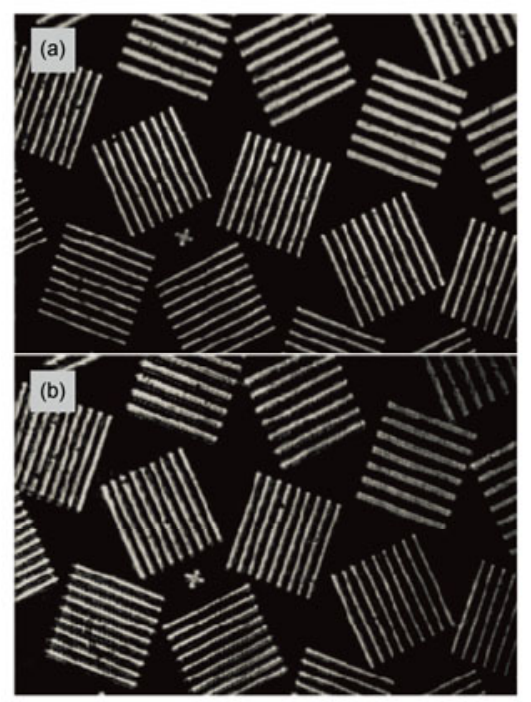

Figure 6 Resolution chart reconstructed with the reference beam (a) after first recording and (b) after 50 write/erase cycles with a negligible decline in resolution.

\section{Conclusion}

A novel diarylethene was synthesized, and its good reversible photochromic behavior had been demonstrated. The absorption spectral changes and kinetics study of the molecule during the photoisomerization showed its rapid response to light. When the molecule was successfully introduced to the field of holographic storage, the maximum refractive index modulation $(0.87 \%$ ) could be reached quickly within just $120 \mathrm{~s}$, which gave the ability of fast recording. The reversible holographic storage ability was also confirmed by the high quality reconstruction after 50 write/erase cycles. All those results indicated that this molecule was a reliable fast write/erase holographic storage material. Besides rewritable holographic recording, this molecule may also be useful in other fields based on its good switching property. Future work will focus on higher resolution storage, super fatigue resistance and higher storage density.

This work was supported by the National Natural Science Foundation of China (21073105), the National Basic Research Program of China (2007CB808002), the National High Technology Research and Development Program of China (2012AA030306) and Tsinghua University Initiative Scientific Research Program (2011Z23149, 2011Z02138).

1 Bruder F K. From the surface to volume: Concepts for the next generation of optical-holographic data-storage. Angew Chem Int Ed, 2011, 50: 4552-4573

2 Bouwhuis G, Braat J, Huijser A, et al. Principles of Optical Disc Systems. Bristol: AdamHilger, 1985

3 McDaniel T W, Victoria R H. Handbook of Magneto-Optical Data Recording. Noyes: Westwood, 1997

4 Watanabe T, Watanabe M. Robust holographic storage system design. Opt Express, 2011, 19: 24147-24158

5 Zeng D, Chen Y. Photochromic diarylethene for rewritable holographic data storage. Opt Express, 2005, 13: 3123-3128

6 Liu G D, He Q S, Ding D H, et al. Diarylethene materials for rewritable volume holographic data storage. Chin Phys Lett, 2003, 20: 1051-1053

7 Wang T, Pu S. Synthesis of a new diarylethene for pptical recording. J Phys: Conf Ser, 2011, 276: 012192

8 Luo S J, Liu G D, He Q S, et al. Holographic grating formation in photochromic diarylethene-doped polymeric thin films. Chin Phys Lett, 2005, 22: 107-109

9 Pu S, Yang T, Yao B, et al. Photochromic diarylethene for polarization holographic optical recording. Mater Lett, 2007, 61: 855-859

10 Pu S, Yao B, Liu G, et al. Polarization multiplexing holographic optical recording of a new photochromic diarylethene. Opt Eng, 2008, 47: 030502

11 Irie M. Diarylethenes for memories and switches. Chem Rev, 2000, 100: 1685-1716

12 Chen Z, Niu L, Cheng Y, et al. Octathienyl/phenyl substituted zinc phthalocyanines $J$-aggregated through conformational planarization. Dalton Trans, 2011, 40: 393-401

13 Fan C, Pu S, Liu G, et al. Substituent position effect on the properties of new unsymmetrical isomeric diarylethenes having a chlorine atom. J Photochem Photobiol A-Chem, 2008, 197: 415-425

14 Kim E, Park J, Cho S Y, et al. Preparation and holographic recording of diarylethene-doped photochromic films. ETRI J, 2003, 25: 253-257

15 Kogelnik H. Coupled wave theory for thick hologram gratings. Bell Syst Tech J, 1969, 48: 2909

Open Access This article is distributed under the terms of the Creative Commons Attribution License which permits any use, distribution, and reproduction in any medium, provided the original author(s) and source are credited. 